\title{
Gradual Correction of Proximal Tibial Biplanar Deformities Using Truelok Hexapod System ${ }^{\mathrm{TM}}$
}

\section{Saleh Alsaifi ${ }^{*}$, Ahmed Abdelaziz ${ }^{2}$, Sameeh Elashry ${ }^{2}$ and Mohamed Mosad $^{2}$}

${ }^{1}$ Head of Pediatric and deformity Unit, Al-Razi Orthopaedic Hospital, Kuwait

${ }^{2}$ Pediatric Unit, Al-Razi Orthopaedic Hospital, Kuwait

*Corresponding Author: Saleh Alsaifi, Head of Pediatric and deformity Unit, Al-Razi Orthopaedic Hospital, Kuwait.
Received: March 29, 2020

Published: April 10, 2020

(c) All rights are reserved by Saleh Alsaifi., et al.

\begin{abstract}
At thirteen-years-old boy who sustained an open physeal injury of the left proximal tibia at four year of age presented to our lower limb deformity clinic complaining from deformity of the left knee and limb length discrepancy. Clinical and radiographic assessment revealed apparent shortening of the left lower limb due to a biplanar deformity of left proximal tibia. The deformity was quantified as a $25^{\circ}$ varus and $20^{\circ}$ procurvatum deformity. The patient underwent treatment in the form of gradual deformity correction and 20 mm over-lengthening with a TL-Hex circular external fixator, along with complete epiphysiodesis of the proximal tibial and fibular physis. Treatment was completed over-six months including correction and consolidation and he returned to full activity one month after frame removal with $20 \mathrm{~mm}$ shoe raise to right lower limb. Normal clinical and radiological alignment of the left lower limb was achieved with good knee and ankle range of motion.
\end{abstract}

Keywords: Biplanar Tibial Deformity; Gradual Correction; Circular Frame; Truelok Hexapod System

\section{Background}

Multiplanar deformities are commonly seen after physeal injuries that result in partial arrest. These injuries frequently result in deformities that have components in both coronal and sagittal planes. Although this suggests that the deformity lies in two planes, it in fact lies in one oblique plane. Apparent limb length discrepancy and short limping gait are expected with sever deformities.

Management of all deformity parameters is necessary with least possible procedures. Although deformity correction of the proximal tibia can often be accomplished with an acute correction and the use of internal fixation, this method has limitations. The presence of poor skin, multiplanar deformities, symptomatic limb length discrepancy or history of infection, lack of postoperative adjustability and potential union problems with opening wedge corrective osteotomy versus further shortening in closing wedge procedure show the limitations of this method.

Correction of deformities can be done using monolateral or circular fixators. While monolateral fixators are more comfortable and less bulky, in many cases, they are not suitable for successful and stable correction. Significant disadvantages of the Ilizarov frame include the need for frame adjustments with creation of additional hinges when correcting multiplanar deformities and problems with accurate calculation of the oblique plane to place hinges with potential translational deformities as a result.
The greatest advantage of Truelok Hexapod systems is the elimination of the need for frame adjustments because they use six struts of adjustable length attached to universal hinges to move an object in six degrees of freedom. Hexapod gradual correction offers a solution of distraction histogenesis of bone and skin beside the ability to correct all deformities simultaneously with minimal invasive procedure.

We report the results of a 13-years-old male who was successfully treated by hexapod gradual correction of a proximal tibial multiplanar deformity.

\section{Case Presentation}

A thirteen-year-old boy presented to our lower limb deformity clinic complaining from deformity and limb length discrepancy of his left lower limb. He was involved in road traffic accident at age of 4 years where he sustained an open physeal injury and massive soft tissue loss over the medial aspect of the left proximal tibia. Treatment at the time on injury consisted of plastic surgery skin graft procedures to the posteromedial aspect of left leg followed by soft tissue release and graft expansion after graft contracture three years later. He was diagnosed after the accident with proximal tibial physis injury and the soft tissue condition at that time did not allow any intervention.

Clinical and radiographic assessment revealed a short limb gait and apparent shortening of the left lower limb due to a biplanar deformity of the left proximal tibia. 
The patient had a short limb gait of left leg, varus attitude (Figure $1 \mathrm{a}$ ) and $20^{\circ}$ procurvatum of the left proximal tibia (Figure $1 \mathrm{~b}$ ). The soft tissue defect over the posteromedial aspect of the left knee was notable (Figure1c) but the skin healed and mobile with no sign of acute or chronic infection and doing well with full flexion of left knee (Figure 1d). No rotational deformity was diagnosed clinically.
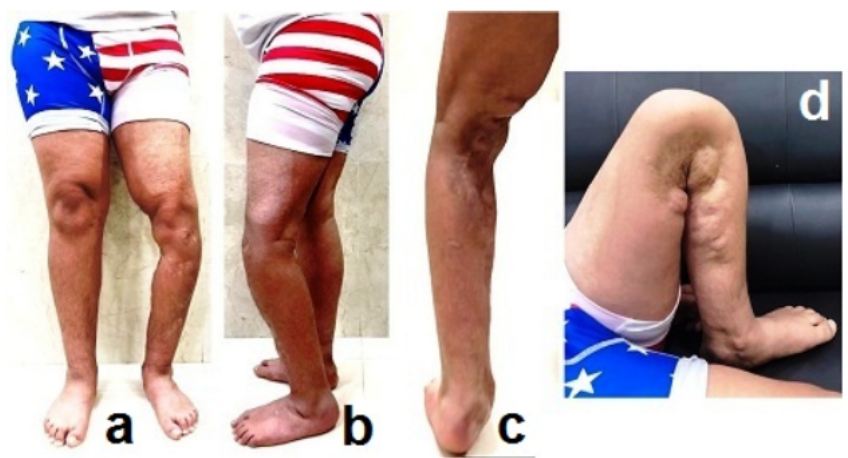

Figure 1: Clinical photos: a) Anterior, b) Lateral side of left knee, c) Posteriorly for left knee and d) Full flexion of left knee.

Radiographic analysis of the long lower limb standing AP and lateral views revealed, mechanical axis deviation (MAD) of 70$\mathrm{mm}$ medial, mechanical lateral distal femoral angle (mLDFA) of $90^{\circ}$, medial proximal tibial angle (MPTA) of $65^{\circ}$ (Figure 2a), posterior proximal tibial angle (PPTA) of $62^{\circ}$ (Figure 2b) [1]. Bilateral segmental analysis of both femora and tibias revealed equal limb lengths.

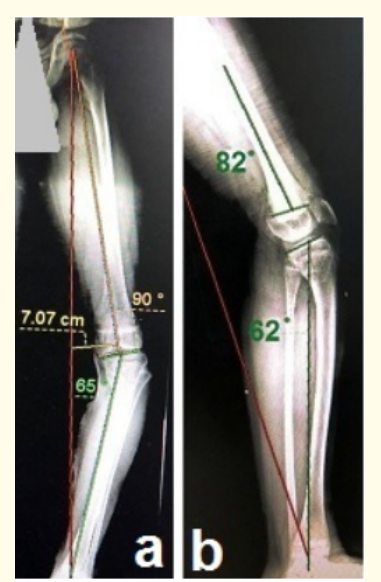

Figure 2: Long leg standing AP X-ray Scanogram

(a) Lateral X-ray Scanogram (b).

The tibial deformity was quantified as $25^{\circ}$ varus and $20^{\circ}$ procurvatum due to posteromedial physeal arrest of proximal tibial physis. The short limb gait was attributed to apparent limb length discrepancy as a result of the severe varus and procurvatum deformities.
Treatment options were discussed with the patient and his family, and as the biplanar deformity of left proximal tibia more than $10^{\circ}$ in both planes the decision was made for gradual correction and lengthening with Truelok Hexapod circular external fixator.

Preoperative planning

1. To prevent recurrence of the deformity, a complete percutaneous epiphysiodesis of both the proximal tibia and fibula was planned.

2. The apex of deformity was located to the proximal tibial metaphyseal region. The tibial percutaneous DeBastiani osteotomy was planned on the transverse bisector line together with a $10 \mathrm{~mm}$ fibula ostectomy at the level of the lower and mid $1 / 3$ of the fibula shaft.

3. To prevent displacement of the distal tibiofibular joint, a percutaneous cannulated screw at the level of the syndesmosis was planned.

4. As the main structure at risk was the posteromedial skin graft, the rate of correction was planned to be less than the customary $1 \mathrm{~mm}$ per day to avoid any soft tissue complications.

5. A preassembled frame was constructed and checked on the patient preoperatively (Figure 3). Preassembled frame technique was used to reduce the time of the theater and optimized struts exchange which was potentially cheaper and more accurate.

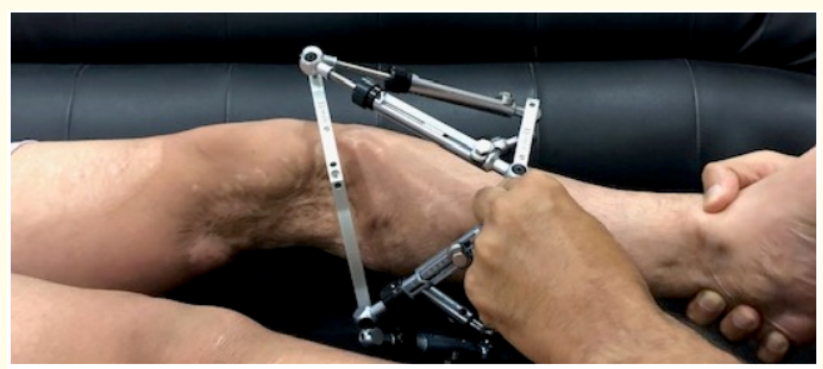

Figure 3: Preassembled frame examination over left leg.

Surgical procedure

1. The surgery was performed under general anesthesia. Prophylactic antibiotic was administered with anesthesia induction. No tourniquet was used during the procedure.

2. Distal tibiofibular joint was fixed with 4.5 cannulated screw and $10 \mathrm{~mm}$ fibular ostectomy was performed as planned (Figure $4 b)$.

3. Complete ablation of lateral side of proximal tibial physis with percutaneous multidirectional drill technique was done then proximal fibular epiphysiodesis was performed (Figure $4 b)$.

4. The prebuilt TL-Hex frame was attached to the bone using one tensioned wire and two perpendicular hydroxyl-apatite (HA) coated half pins on each ring (Figure 4a and 4b) [2]. A 5/8th ring was used proximally to accommodate greater knee flexion. 
5. The multiple drill hole DeBastiani osteotomy of the tibia was performed and completed with an osteotome. Final frame mounting parameters were calculated after placement of the frame.

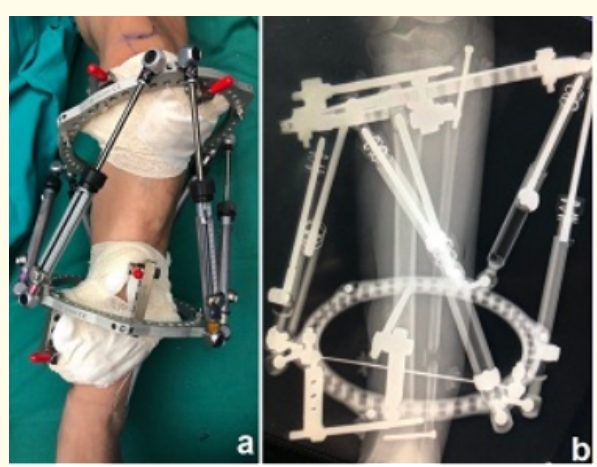

Figure 4: Clinical photo on operation table (a), X-ray full AP left tibia (b).

\section{Rehabilitation}

After surgery, the patient was allowed to bear weight as tolerated (Figure 5) and range of motion exercises for knee and ankle were encouraged. A daily shower, including washing the pin sites with antibacterial soap, was encouraged one week after the surgery. This was followed by twice daily pin care with Chlorhexidine $0.5 \%$ in water.

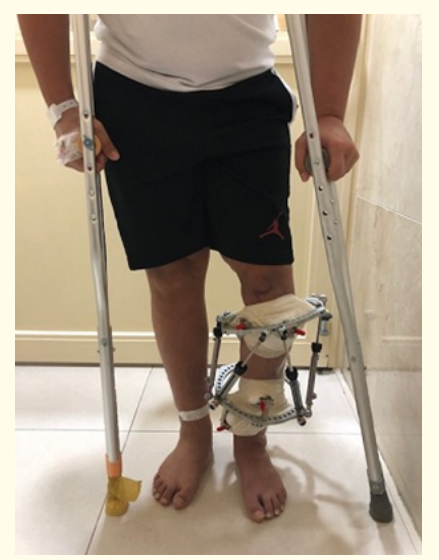

Figure 5: Day 1 postoperative, partial weight bearing left leg mobilization with crutches.

Tibial deformity parameters of $25^{\circ}$ varus, $20^{\circ}$ procurvatum and $20 \mathrm{~mm}$ desired lengthening was entered into the TL-Hex webbased software to generate an adjustment schedule (Figure 6).

The program required input of deformity, frame, mounting parameters, and determine the rate of correction. A latency period of 7 days was observed after which deformity correction was commenced. The patient was discharged with instructions to weight bear as tolerated with crutches. Radiographs taken at 2 weeks confirmed that the osteotomy was complete, and the correction was in progress.
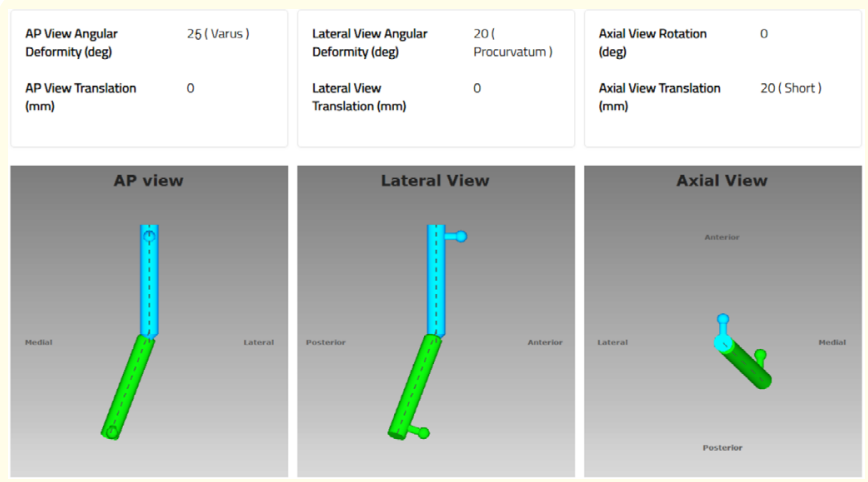

Figure 6: Deformity parameter entered into the TL-Hex web-based software.
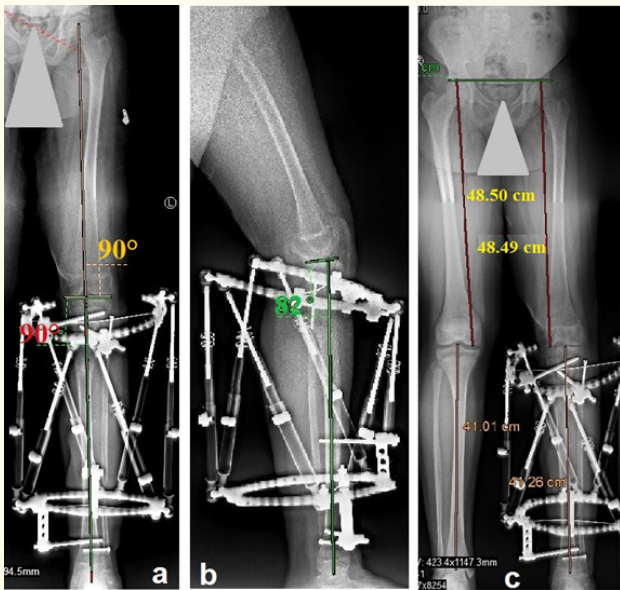

Figure 7: Long leg standing X-ray Scanogram; a) AP view after varus correction MAD $=0 \mathrm{~mm}$ and MPTA $=90^{\circ}$, b) Lateral view after procurvatum correction PPTA $=82^{\circ}$, c) Segmental length for both sides after angular correction and before left tibial lengthening.

Weekly clinic visits during the correction phase (8 weeks) was changed to monthly visits once alignment was achieved and continued until frame removal. At the end of correction, the limb alignment was determined with physical radiographic analysis. On long lower limb standing radiograph, we measured MAD, MPTA, PPTA and LLD (Figure 7) using the same methods used before surgery, we assessed the rotation by patella up test and TFA. A second program was generated to correct minor residual deformity.

Frame removal was considered once the ability to walk with minimal assistance and no pain at the osteotomy site was achieved clinically. The presence of bridging callus on three of the four cortices on the anteroposterior, internal oblique, external oblique and lateral radiographs was assessed prior to frame removal.

\section{Result}

Clinically the left knee appeared in straight position (Figure 8d) with good range of motion. The foot was in plantigrade position. 
At completion of treatment (Figure 8a); the MAD decreased from $70 \mathrm{~mm}$ to $0 \mathrm{~mm}$, MPTA corrected from $65^{\circ}$ to $90^{\circ}$, and the length of the left tibia was increased by $20 \mathrm{~mm}$ (Figure 8c). In the sagittal plane, the PPTA was corrected from $62^{\circ}$ to $82^{\circ}$ (Figure $8 \mathrm{~b}$ ).

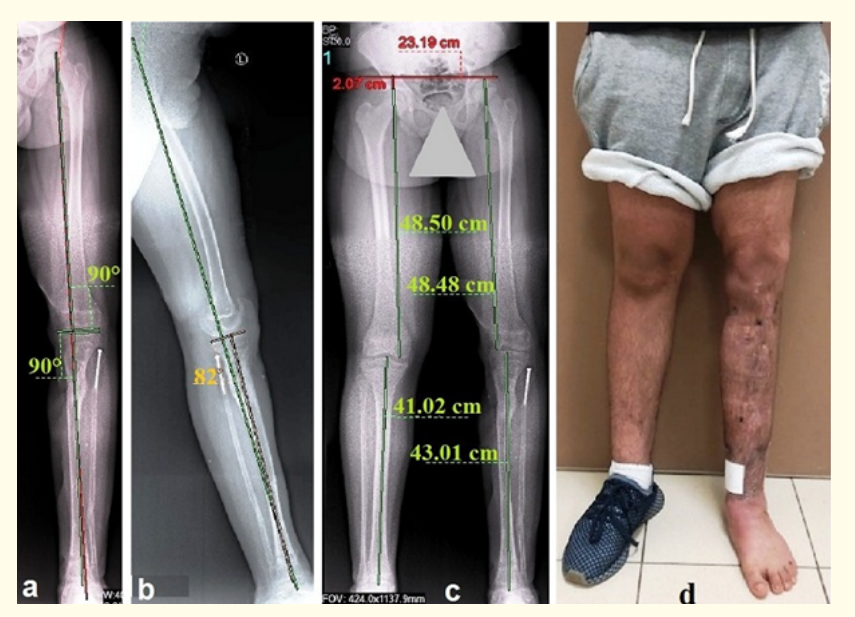

Figure 8: Long leg standing X-ray Scanogram after removal of Truelok Hexapod Frame, a) Aligned left knee: $\operatorname{mLDFA}=90^{\circ}$, MPTA $=90^{\circ}$ and MAD $=0 \mathrm{~mm}, \mathrm{~b}$ ) Full extension left knee PPTA $=82^{\circ}, \mathrm{c}$ ) Left tibia longer with $20 \mathrm{~mm}$ than right tibia, d) Clinical photo after frame removal showing straight left knee.

Our aim of precise correction of coronal and sagittal plane deformities was achieved along with $20 \mathrm{~mm}$ tibial lengthening to compensate for expected growth of the right leg.

\section{Discussion and Conclusion}

Although deformity correction of the proximal tibia can often be accomplished with an acute correction and the use of internal fixation, this method has limitations [3,4]. The presence of poor skin, multiplanar deformities, symptomatic limb length discrepancy or history of infection and lack of postoperative adjustability show the limitations of this method.

The Ilizarov method of deformity correction and limb lengthening was the most important contribution in the field of deformity correction in the last century [5]. Significant disadvantages of the Ilizarov frame include a steep learning curve and the need for frame adjustments with creation of additional hinges when correcting multiplanar deformities.

Hexapod systems has eliminated the need for frame adjustments because of their use of six adjustable struts attached to universal hinges to move an object in six degrees of freedom. The TL-Hex circular external fixator is based on identical biological properties and host response as those of a traditional Ilizarov fixator, with possibly better mechanical properties and ease of use [6].

The TL-Hex circular external fixator is able to correct six-axis deformities simultaneously with great accuracy. Therefore, after application of the frame all that is left for the surgeon to do is to perform accurate deformity analysis and insert correct data to the website program. Undoubtedly, hexapod system has become the treatment of choice for multiplanar skeletal deformities.

\section{Conflict of Interest}

No conflict of interest exists.

\section{Bibliography}

1. Paley D. "Normal lower limb Alignment and Joint Orientation". In: Paley D, editor. Principles of Deformity Correction, $1^{\text {st }}$ edition. Berlin, Germany: Springer-Verlag (2005): 11-17.

2. Pizà G., et al. "Hydroxyapatite-coated external-fixation pins. The effect on pin loosening and pin track infection in leg lengthening for short stature". Journal of Bone and Joint Surgery 86 (2004): 892-897.

3. Adili A., et al. "Valgus high tibial osteotomy. Comparison between an Ilizarov and a Coventry wedge technique for the treatment of medial compartment osteoarthritis of the knee". Knee Surgery, Sports Traumatology, Arthroscopy 10 (2002): 169-176.

4. Sprenger TR and Doerzbacher JF. "Tibial osteotomy for the treatment of varus gonarthrosis. Survival and failure analysis to twenty-two years". Journal of Bone and Joint Surgery: American Volume 85.3 (2003): 469-474.

5. Ilizarov GA. "Clinical application of the tension-stress effect for limb lengthening". Clinical Orthopaedics and Related Research 250 (1990): 8-26

6. Paley D. "Six-axis deformity analysis and correction". In: Paley D, editor. Principles of Deformity Correction, $1^{\text {st }}$ edition. Berlin, Germany: Springer-Verlag (2005): 418-436.

\section{Assets from publication with us}

- Prompt Acknowledgement after receiving the article

- Thorough Double blinded peer review

- Rapid Publication

- Issue of Publication Certificate

- High visibility of your Published work

Website: https://www.actascientific.com/ Submit Article: https://www.actascientific.com/submission.php Email us: editor@actascientific.com Contact us: +919182824667 